\title{
Interactive comment on "Parameterizing anisotropic reflectance of snow surfaces from airborne digital camera observations in Antarctica" by Tim Carlsen et al.
}

\section{Anonymous Referee \#2}

Received and published: 3 July 2020

The paper describes an approach for characterizing snow directional reflectance using airborne data (spectral surface albedo measurements, and surface roughness determined by means of a laser scanner) that were taken over a large area $(1000 \times 1000$ km2) located in the East Antarctic Plateau during austral summer in 2013/14 using a single channel $(490-585 \mathrm{~nm})$ digital $180^{\circ}$ fish-eye camera. The study provides new parameterization of the semi-empirical kernel-driven bidirectional reflectance model (RossThick-LiSparse-Reciprocal (RTLSR)), which is used in the operational MODIS BRDF/albedo product retrieval, based on the airborne data of snow BRDF as a function of solar zenith angle, surface roughness, and optical-equivalent snow grain size. 
1. Section 1: provides an excellent background literature review of snow reflectance anisotropy, snow BRDF models and verification of the models with existing BRDF measurements.

2. Section 2: Methodology - the definitions of reflectance quantities are articulated well, however, the use of symbols is inconsistent. For example, in subsection 2.1, the symbol for the solar zenith angle is given as theta(i) (see Equation 1), then the subscript changes to something else, theta(0) (see Equation 3) without any explanation. In subsection 2.2, the symbol for the relative azimuth angle are different both in Equation 4 and Equation 7.

3. Subsection 3.2 - optical-equivalent grain size retrieval from spectral surface albedo measurements. It was reported elsewhere in the manuscript (subsection 3.4) that "the downward irradiance measurements from SMART could not be used for the calculation of the HDRF due to calibration issues." So does this imply that the optical-equivalent snow grain size retrieved from SMART measurements may be affected by those calibration issues? It's also odd that the "optical-equivalent snow grain size from SMART and analogous ground-based measurements were validated against grain size observations utilizing reflectance measurements with MODIS." Which retrievals are assumed to be the "truth"?

4. Subsection 3.3.2 - Radiance calibration and image post-processing. This subsection provides important details about the post processing, but there are no details about the pre-deployment calibration. Given that the flights were performed between $24 \mathrm{De}$ cember 2013 and 5 January 2014, it may be shown that each flight need a different calibration, which would depend on the calibration stability of the digital camera Canon EOS-1D Mark III.

Specific comments: 1. Pg. 1. Line 17: The study finds that "MODIS observations generally underestimated the anisotropy of the surface reflection", but it would help to

Printer-friendly version

Discussion paper

2 
provide the degree to which MODIS underestimates the anisotropy of surface reflection.

2. Pg. 2, Line 4: The statement, "Satellites monitor the reflectance (i.e., reflected radiance in units of $\mathrm{W} \mathrm{m}-2 \mathrm{sr}-1$ ) at the top of the atmosphere (TOA). However, they are restricted in terms of the number of available observation angles and spectral bands as well as their temporal resolution." Is this in reference to polar orbiting satellites or geostationary satellites?

3. Pg. 2, Line 8, the statement, "During the first step, the TOA reflectance is converted into a surface reflectance by means of an atmospheric radiative transfer parameterization," need to be clarified. What is "atmospheric radiative transfer parameterization?"

4. Pg. 2, line 10, the recommended terminology: bidirectional reflectance-distribution function (BRDF). Note the "reflectance-distribution" is a compound adjective and should be hyphenated.

5. Pg. 3, line 19, change the statement, "Comparing this asymptotic model to in situ observations of the BRDF, ..." to "Evaluating this asymptotic model with in situ observations of the BRDF, ..."

6. Pg. 3, in the paragraph, marked by lines $8-25$, various snow reflectance terms are mentioned: snow BRDF (line 10), a polarized BRDF (line 12), reflectance of snow (line 18 ), inherent optical properties (line 22), and bidirectional reflectance (line 24). This may be confusing to readers and it would help to clarify or use consistent terms.

7. Pg. 3, line 29, the use of "solar azimuth" and "azimuthal directions" in this sentence is confusing, "Kuhn (1985) observed a peak in reflectance in the azimuthal directions up to $60^{\circ}$ to both sides of the solar azimuth that becomes more prominent with increasing solar zenith angle and snow grain size." What is the zero azimuth in this case?

8. Pg. 4, line 28 , change "480" to "340". The smallest CAR wavelength is $340 \mathrm{~nm}$.

9. Pg. 5, line 29, the BRDF symbol used here is not consistent with Schaepman-Strub 
et al. (2006) as stated/line 27.

10. Pg. 5, line 30 , the statement "the reflected radiance for all reflection angles ..." is unclear. The entire definition of the spectral BRDF need to be clarified.

11. Pg. 13 , line 14 , the location of each pixel is given by $x$ and $y$, but " $x$ " and " $y$ " are not defined elsewhere in the manuscript.

12. Pg. 6, the derivation of Equation (3) is not clear.

13. Pg. 13, line 19, the author need to show how "... the viewing angles are corrected" using the "the roll, pitch and yaw angles of the aircraft at the time of measurement."

14. Pg. 13, Equation 18, the viewing and illumination geometry variables need to be defined taking into account the aircraft orientation. The downward flux $\mathrm{F}$ should also be described here immediately after the equation is listed, unless it's previously defined.

Interactive comment on The Cryosphere Discuss., https://doi.org/10.5194/tc-2020-97, 2020. 